\title{
A nomogram predicting overall survival in patients with non-metastatic pancreatic head adenocarcinoma after surgery: a population-based study
}

\author{
Wenbo Zou ${ }^{1,2,3+}$, Zizheng Wang ${ }^{1,2,3+}$, Fei Wang ${ }^{1,2,3 \dagger}$, Gong Zhang ${ }^{1,2,3}$ and Rong Liu ${ }^{1,2,3^{*}}$
}

\begin{abstract}
Background: Pancreatic head adenocarcinoma (PHAC), a malignant tumour, has a very poor prognosis, and the existing prognostic tools lack good predictive power. This study aimed to develop a better nomogram to predict overall survival after resection of non-metastatic PHAC.

Methods: Patients with non-metastatic PHAC were collected from the Surveillance, Epidemiology, and End Results (SEER) database and divided randomly into training and validation cohorts at a ratio of 7:3. Cox regression analysis was used to screen prognostic factors and construct the nomogram. Net reclassification improvement (NRI) and integrated discrimination improvement (IDI) were calculated to evaluate the performance of the model. The predictive accuracy and clinical benefits of the nomogram were validated using the area under the curve (AUC), calibration curves, and decision curve analysis (DCA).

Results: From 2010 to 2016, 6419 patients with non-metastatic PHAC who underwent surgery were collected from the SEER database. A model including T stage, N stage, grade, radiotherapy, and chemotherapy was constructed. The concordance index of the nomogram was 0.676 , and the AUCs of the model assessing survival at multiple timepoints within 60 months were significantly higher than those of the American Joint Committee on Cancer (AJCC) 8th staging system in the training cohort. Calibration curves showed that the nomogram had ability to predict the actual survival. The NRI, IDI, and DCA curves also indicated that our nomogram had higher predictive capability and clinical utility than the AJCC staging system.

Conclusions: Our nomogram has an ability to predict overall survival after resection of non-metastatic PHAC and includes prognostic factors that are easy to obtain in clinical practice. It would help assist clinicians to conduct personalized medicine.
\end{abstract}

Keywords: Pancreatic head adenocarcinoma, Nomogram, Overall survival, SEER

\footnotetext{
* Correspondence: liurong301@126.com

tWenbo Zou, Zizheng Wang and Fei Wang contributed equally to this work.

${ }^{1}$ Faculty of Hepato-Pancreato-Biliary Surgery, Chinese PLA General Hospital,

No.28 Fuxing Road, Haidian District, Beijing 100853, China

${ }^{2}$ Institute of Hepatobiliary Surgery of Chinese PLA, Beijing, China

Full list of author information is available at the end of the article
}

(c) The Author(s). 2021 Open Access This article is licensed under a Creative Commons Attribution 4.0 International License, which permits use, sharing, adaptation, distribution and reproduction in any medium or format, as long as you give appropriate credit to the original author(s) and the source, provide a link to the Creative Commons licence, and indicate if changes were made. The images or other third party material in this article are included in the article's Creative Commons licence, unless indicated otherwise in a credit line to the material. If material is not included in the article's Creative Commons licence and your intended use is not permitted by statutory regulation or exceeds the permitted use, you will need to obtain permission directly from the copyright holder. To view a copy of this licence, visit http://creativecommons.org/licenses/by/4.0/ The Creative Commons Public Domain Dedication waiver (http://creativecommons.org/publicdomain/zero/1.0/) applies to the data made available in this article, unless otherwise stated in a credit line to the data. 


\section{Background}

Pancreatic cancer has a high mortality rate, and the average 5-year survival rate in the United States is approximately $10 \%$ [1]. Because the early symptoms are not obvious, pancreatic cancer is difficult to diagnose and has poor treatment outcomes, which also increase the mortality rate $[1,2]$. Currently, radical surgery and adjuvant therapy including radiotherapy and chemotherapy are the main treatment strategies for pancreatic cancer, and radical surgery is the only curative treatment [3, 4]. However, few patients are suitable for surgical treatment because of distant metastases or local invasion at the time of diagnosis $[5,6]$.

The location of the primary tumour can also affect prognosis and overall survival (OS), and pancreatic head adenocarcinoma (PHAC) is reported to have a significantly shorter OS relative to pancreatic bodytail [7]. Assessment of tumour prognosis primarily relies on the American Joint Committee on Cancer (AJCC) 8th edition staging system [8], which is based on the size and extent of the tumour, the number of metastatic lymph nodes, and the number of distant metastases. The AJCC staging system can guide clinical practice and prognostic analysis to some extent [9]. However, some important prognostic factors are not taken into account in the AJCC staging system, such as age at diagnosis and tumour grade, which reduces its predictive accuracy for some patients. Several prognostic models have been developed to predict the prognosis of pancreatic body/tail tumours [10, 11], although models of non-metastatic pancreatic head tumours still deserve to be explored further. Therefore, a nomogram is needed to predict survival of patients after resection of non-metastatic PHAC and assist with treatment decision-making.

In this study, we screened significant independent factors from the Surveillance, Epidemiology, and End Results (SEER) database to develop a nomogram for modifying the AJCC 8th staging system using Cox regression analysis. The nomogram was constructed for predicting survival in a training cohort and verified in a validation cohort. This nomogram would help assist clinicians to conduct personalized medicine.

\section{Methods}

\section{Study population and selection criteria}

Patient information was collected from the SEER database of the National Cancer Institute (http://seer.cancer. gov/). After consulting the CS Schema v0204+, we extracted data from 115,227 patients with pancreatic head tumours using SEER*Stat software (version 8.3.8). The inclusion criteria were as follows: (a) International Classification of Diseases for Oncology, Third Edition (ICDO-3) histology codes 8140, 8255, 8480, 8481, 8500, 8503, 8521, 8523, and 8527; (b) ICD site code C25.0; (c) M0 stage; (d) survival time $>30$ days; (e) age $\geq 20$ years; (f) underwent surgery; and (g) complete information on race, sex, grade, AJCC staging, radiotherapy, chemotherapy, and the number and status of lymph nodes. Patient lacking any of this information were excluded from the study cohort. Because the original SEER cohort used the 7th edition AJCC staging, we transformed the 7th edition staging to the 8th edition staging for subsequent analysis based on the information about the size and extent of the tumour and the number of positive lymph nodes. The included patients were divided randomly into a training cohort $(n=4495)$ and a validation cohort $(n=$ 1924) at a ratio of 7:3 using the 'caret' package in $R$. The training cohort was used to select the prognostic factors and construct the model, and the model performance was verified in the validation cohort. A detailed datascreening process is shown in Fig. 1.

\section{Statistical analysis}

Continuous variables are reported as median (interquartile range) and were analysed by Student's t test, and categorical variables are reported as counts and proportions and were analysed by chi-squared test for comparisons among groups. Identification of the independent prognostic factors and development of the nomogram model predicting OS was implemented using univariate and multivariate Cox regression analysis. The optimal cut-off values of the lymph node ratio (LNR), tumour size, and total risk score were calculated via X-tile (version 3.6.1). The LNR was defined as the ratio of the number of positive lymph nodes to the number of total examined lymph nodes. We converted continuous variables to categorical variables for further processing and divided patients into 'low', 'medium', and 'high' groups in the training cohort based on the optimal threshold of the total risk score. OS was the primary endpoint and was defined as the time from diagnosis to death or the last follow-up. Kaplan-Meier (KM) survival analysis was performed to depict the capability of individual variables to discriminate OS. With the aim of predicting the OS of patients, a nomogram was finally constructed. The concordance index (C-index) was calculated to evaluate the discrimination of the model, and calibration curves and time-dependent receiver operating characteristic curves within 60 months were created to estimate the predictive capability of the model. The net reclassification improvement (NRI) and integrated discrimination improvement (IDI) were calculated to evaluate the improvement of the model prediction compared to the 8th AJCC staging using the 'nricens' and 'rms' packages. Decision curve analysis (DCA) is widely applied to evaluate a nomogram with the advantage of integrating patients' or decisionmakers' preferences into the analysis and is increasingly used in clinical studies. We performed DCA to analyse 


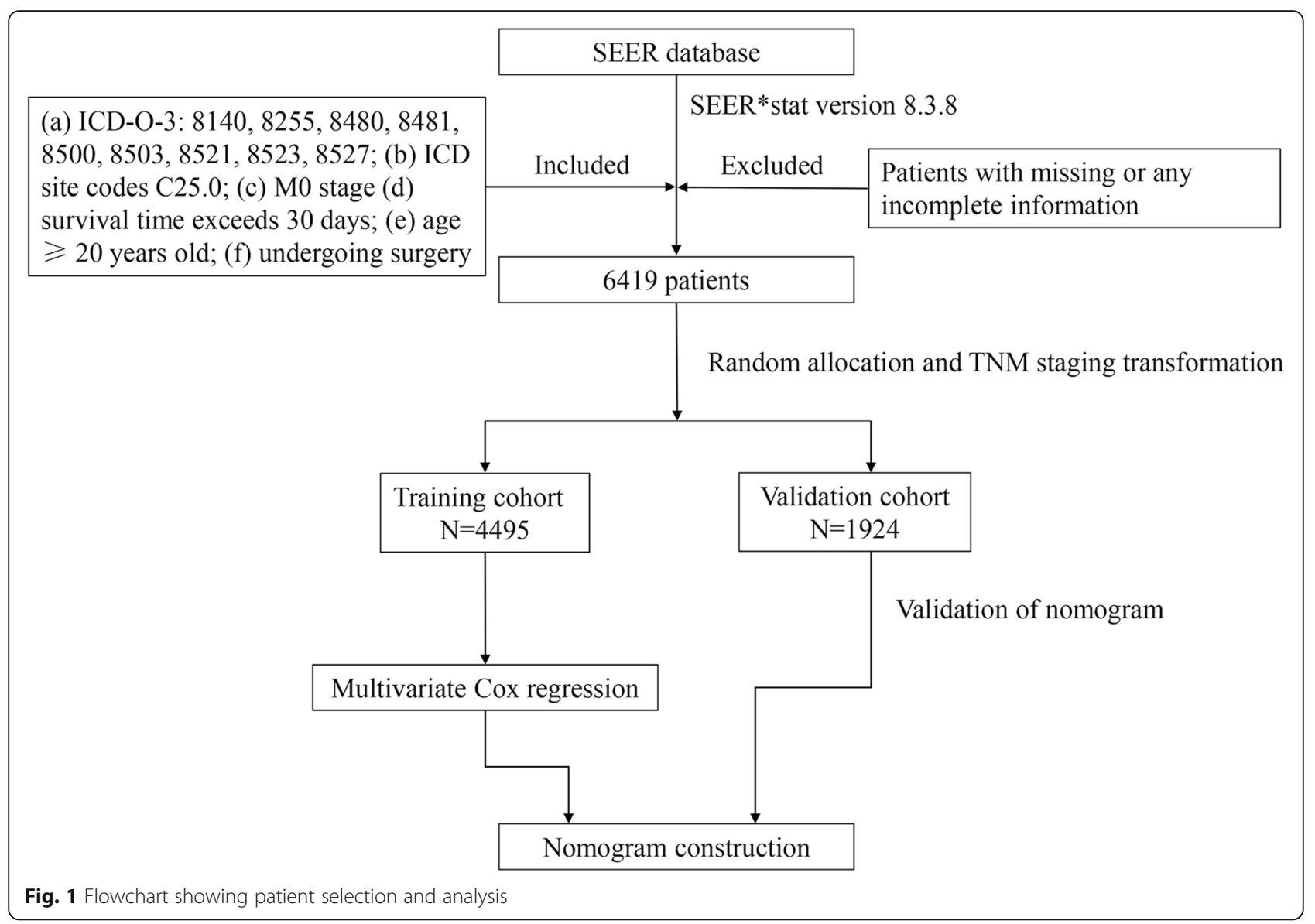

the clinical benefits of the nomogram compared with those of the 8th AJCC staging system. R (version 4.0.2) and SPSS (version 26.0) were used to complete all statistical analyses in our study, and the main utilized $\mathrm{R}$ packages were 'nricens', 'Cschange', 'rms', and 'timeROC'. A two-sided $p<0.05$ was considered statistically significant.

\section{Results}

\section{Demographics and characteristics of the included} patients

A total of 6419 patients who underwent resection for non-metastatic PHAC met the criteria and were included in the analysis. Based on the 7:3 ratio of distribution, 4495 and 1924 patients were sorted into the training and validation cohorts, respectively. The median OS in these two cohorts were $16(8-28)$ months and 16 (9-27) months, respectively. In the training cohort, most of the patients were $60-80$ years old (2984 [66.4\%]), the male $(2269$ [50.5\%]) to female $(2226$ [49.5\%]) ratio was $1.02: 1$, and the predominant race was white (3712 [82.6\%]). In terms of pathological diagnosis, most of the patients had T2 stage disease (2798 [62.2\%]), N1 stage disease (1823 [40.6\%]), and moderately differentiated tumours $(2355$ [52.4\%]). Stage II was the most common clinical stage (1931 [43.0\%]). Most patients had tumours $>2.5 \mathrm{~cm}$ (2948 [65.6\%]). Further, most patients received chemotherapy (3362 [74.8\%]) and did not receive radiotherapy (3054 [67.9\%]). The median number of retrieved lymph nodes was 17 (12-24), and the median number of positive lymph nodes was $2(0-4)$.

To better guide clinical practice, two continuous variables in the training cohort, the LNR and tumour size, were stratified via X-tile (Supplementary Fig. S1 and Table S1). The LNR ranged from 0 to 1 , and the tumour size ranged from $0.1 \mathrm{~cm}$ to $46 \mathrm{~cm}$. The LNR was grouped into LNR1 (LNR $\leq 0.20)$ and LNR2 (LNR >0.20), and tumour size was classified into group 1 (diameter $\leq 2.5$ $\mathrm{cm}$ ) and group 2 (diameter $>2.5 \mathrm{~cm}$ ). The baseline clinicopathological characteristics, demographic characteristics, and comparable $p$ values are shown in Table 1.

\section{Identification and selection of prognostic factors}

We performed Cox regression analysis to identify significant prognostic factors correlated with OS. As shown in Table 2, univariate regression analysis revealed that clinicopathological characteristics, including age, grade, $\mathrm{T}$ stage, $\mathrm{N}$ stage, the LNR, and tumour size, were prognostic risk factors $(p<0.001)$, whereas interventions, 
Table 1 Demographics and characteristics of patients in Training and Validation cohorts

\begin{tabular}{|c|c|c|c|}
\hline Characteristics & $\begin{array}{l}\text { Training cohort } \\
(n=4495)\end{array}$ & $\begin{array}{l}\text { Validation cohort } \\
(n=1924)\end{array}$ & $\begin{array}{l}p \\
\text { value }\end{array}$ \\
\hline Age (year) & n (\%) & n (\%) & 0.070 \\
\hline$<40$ & $38(0.8 \%)$ & $6(0.3 \%)$ & \\
\hline $40-60$ & 1010 (22.5\%) & $454(23.6 \%)$ & \\
\hline $60-80$ & 2984 (66.4\%) & $1280(66.5 \%)$ & \\
\hline$\geq 80$ & $463(10.3 \%)$ & $184(9.6 \%)$ & \\
\hline Gender & & & 0.102 \\
\hline Female & 2226 (49.5\%) & $910(47.3 \%)$ & \\
\hline Male & $2269(50.5 \%)$ & 1014 (52.7\%) & \\
\hline Race & & & 0.413 \\
\hline White & 3712 (82.6\%) & 1596 (83.0\%) & \\
\hline Black & 411 (9.1\%) & 186 (9.6\%) & \\
\hline Others & 372 (8.3\%) & $142(7.4 \%)$ & \\
\hline 8th T stage & & & 0.100 \\
\hline $\mathrm{T} 1$ & 739 (16.4\%) & $293(15.2 \%)$ & \\
\hline $\mathrm{T} 2$ & 2798 (62.2\%) & 1195 (62.1\%) & \\
\hline T3 & 786 (17.5\%) & $376(19.6 \%)$ & \\
\hline T4 & $172(3.8 \%)$ & 60 (3.1\%) & \\
\hline 8th N stage & & & 0.931 \\
\hline NO & $1321(29.4 \%)$ & $561(29.1 \%)$ & \\
\hline $\mathrm{N} 1$ & $1823(40.6 \%)$ & 790 (41.1\%) & \\
\hline N2 & 1351 (30.0\%) & $573(29.8 \%)$ & \\
\hline Grade & & & 0.086 \\
\hline Well & $476(10.6 \%)$ & $222(11.5 \%)$ & \\
\hline Moderate & 2355 (52.4\%) & 1005 (52.2\%) & \\
\hline Poor & 1638 (36.4\%) & $676(35.2 \%)$ & \\
\hline Undifferentiated & $26(0.6 \%)$ & $21(1.1 \%)$ & \\
\hline LNR & & & 0.590 \\
\hline 1 & 3086 (68.7\%) & 1334 (69.3\%) & \\
\hline 2 & $1409(31,3 \%)$ & $590(30.7 \%)$ & \\
\hline Tumor size $(\mathrm{cm})$ & & & 0.909 \\
\hline$\leq 2.5$ & 1547 (34.4\%) & 665 (34.6\%) & \\
\hline$>2.5$ & $2948(65.6 \%)$ & $1259(65.4 \%)$ & \\
\hline Radiotherapy & & & 0.647 \\
\hline No/unkown & 3054 (67.9\%) & $1296(67.4 \%)$ & \\
\hline Yes & 1441 (32.1\%) & $628(32.6 \%)$ & \\
\hline Chemotherapy & & & 0.999 \\
\hline No/unkown & 1133 (25.2\%) & 485 (25.2\%) & \\
\hline Yes & $3362(74.8 \%)$ & $1439(74.8 \%)$ & \\
\hline 8th AJCC stage & & & 0.749 \\
\hline Stage I & $1100(24.5 \%)$ & $460(23.9 \%)$ & \\
\hline Stage II & 1931 (43.0\%) & $846(44.0 \%)$ & \\
\hline Stage III & $1464(32.5 \%)$ & $618(32.1 \%)$ & \\
\hline RLNs & 17 [12-24] & 17 [11.25-23] & 0.409 \\
\hline PLNs & $2[0-4]$ & $2[0-4]$ & 0.689 \\
\hline mOS (months) & 16 [8-28] & 16 [9-27] & 0.539 \\
\hline
\end{tabular}

LNR lymph node ratio, RLNs retrieved lymph nodes, PLNs positive lymph nodes, mOS median overall survival 
Table 2 Univariate Cox analysis of prognostic factors in the training cohort

\begin{tabular}{|c|c|c|c|c|c|}
\hline \multirow[t]{2}{*}{ Variables } & \multirow{2}{*}{$\begin{array}{l}\text { No. of patients } \\
\mathrm{N}\end{array}$} & \multirow{2}{*}{$\begin{array}{l}\text { Overall survival } \\
\mathrm{mOS} \text { [IQR] }\end{array}$} & \multicolumn{3}{|c|}{ Univariate analysis } \\
\hline & & & $\mathrm{HR}$ & $95 \% \mathrm{Cl}$ & $p$ value \\
\hline Age & & & 1.24 & $1.16-1.32$ & $<0.001$ \\
\hline$<40$ & 38 & 26 [19-35] & & & \\
\hline $40-60$ & 1010 & $23[21-25]$ & & & \\
\hline $60-80$ & 2984 & $21[20-22]$ & & & \\
\hline$\geq 80$ & 463 & 15 [13-18] & & & \\
\hline Gender & & & 1.03 & $0.96-1.11$ & 0.460 \\
\hline Female & 2226 & 21 [20-22] & & & \\
\hline Male & 2269 & 21 [20-22] & & & \\
\hline Race & & & 1.02 & $0.96-1.08$ & 0.576 \\
\hline White & 3712 & $21[20-22]$ & & & \\
\hline Black & 411 & 22 [18-25] & & & \\
\hline Others & 372 & 20 [18-23] & & & \\
\hline Grade & & & 1.45 & $1.37-1.54$ & $<0.001$ \\
\hline Well & 476 & $32[27-36]$ & & & \\
\hline Moderate & 2355 & 24 [22-25] & & & \\
\hline Poor & 1638 & 16 [15-17] & & & \\
\hline Undifferentiated & 26 & 18 [11-26] & & & \\
\hline 8th T stage & & & 1.31 & $1.25-1.38$ & $<0.001$ \\
\hline $\mathrm{T} 1$ & 739 & 31 [27-34] & & & \\
\hline $\mathrm{T} 2$ & 2798 & $21[20-22]$ & & & \\
\hline T3 & 786 & 16 [15-17] & & & \\
\hline T4 & 172 & 16 [16-20] & & & \\
\hline 8th N stage & & & 1.44 & $1.37-1.51$ & $<0.001$ \\
\hline NO & 1321 & 30 [28-33] & & & \\
\hline N1 & 1823 & 20 [19-22] & & & \\
\hline N2 & 1351 & 16 [15-17] & & & \\
\hline Tumor size & & & 1.5 & $1.39-1.63$ & $<0.001$ \\
\hline Diameter $\leq 2.5$ & 1547 & 26 [24-29] & & & \\
\hline Diameter $>2.5$ & 2948 & 18 [18-20] & & & \\
\hline LNR & & & 1.82 & $1.68-1.96$ & $<0.001$ \\
\hline 1 & 3086 & 24 [24-25] & & & \\
\hline 2 & 1409 & 15 [14-16] & & & \\
\hline Radiotherapy & & & 0.71 & $0.65-0.77$ & $<0.001$ \\
\hline No/unkown & 3054 & 19 [18-20] & & & \\
\hline Yes & 1441 & 25 [23-27] & & & \\
\hline Chemotherapy & & & 0.52 & $0.48-0.56$ & $<0.001$ \\
\hline No/unkown & 1133 & 12 [11-13] & & & \\
\hline Yes & 3362 & 24 [23-25] & & & \\
\hline
\end{tabular}

mOS: median overall survival; IQR: interquartile range; HR: Hazard Ratio; Cl: Confidence Interval; LNR: lymph node ratio

including radiotherapy and chemotherapy, were prognostic protective factors $(p<0.001)$. There was no difference in prognosis based on race or gender. The corresponding KM survival curves exhibited the capability of these variables to discriminate the OS in the training cohort (Supplementary Fig. S2).

To improve the application of the model, we further reduced the model variables. $\mathrm{T}$ stage is based on tumour 
size and extension, and the LNR and $\mathrm{N}$ stage both describe the metastatic lymph node status. Therefore, we compared the $\mathrm{C}$-indexes of $\mathrm{T}$ stage and tumour size and those of the LNR and $\mathrm{N}$ stage in the training cohort. The C-index of $\mathrm{T}$ stage was 0.556 , which was superior to that of tumour size $(0.545, p=0.015)$, and the C-index of $\mathrm{N}$ stage was 0.578 , which was superior to that of the LNR $(0.569, p=0.026)$. Therefore, we finally developed a better model composed of $\mathrm{T}$ stage, $\mathrm{N}$ stage, grade, radiotherapy, and chemotherapy. The detailed multivariate Cox regression results are shown in Table 3.

\section{Development and validation of the nomogram predicting overall survival}

Based on this multivariate model, a visualized nomogram was constructed to predict the 1-, 2-, 3-, and 5-year survival rates (Fig. 2). The C-index of the nomogram was 0.676 (95\% CI 0.665-0.687), and it had better predictive capability than the AJCC 8th staging system (C-index: $0.581,95 \%$ CI $0.570-0.591, p<0.001)$ in the training cohort. Similarly, in the validation cohort, the $\mathrm{C}$-index was 0.667 (95\% CI 0.649-0.684), and it also had better predictive capability than the AJCC 8th staging system in this cohort (C-index: 0.566, 95\% CI 0.548-0.584, $p<0.001$ ). The NRI and IDI were also calculated to demonstrate the predictive accuracy of the nomogram. In the training cohort, the NRIs based on bootstrapping for OS at 1, 2, 3, and 5 years were 0.305 (95\% CI 0.248-0.357), 0.227 (95\% CI $0.172-0.284$ ), 0.057 (95\% CI $0.003-0.108$ ), and 0.132

Table 3 Multivariate Cox analysis of prognostic factors in the training cohort

\begin{tabular}{llll}
\hline \multirow{2}{*}{ Variables } & \multicolumn{3}{l}{ Multivariate analysis } \\
\cline { 2 - 4 } 8th T stage & HR & $\mathbf{9 5 \% C l}$ & $\boldsymbol{p}$ value \\
T1 & Reference & & \\
T2 & 1.43 & $1.28-1.60$ & $<0.001$ \\
T3 & 1.79 & $1.57-2.05$ & $<0.001$ \\
T4 & 2.05 & $1.67-2.52$ & $<0.001$ \\
8th N stage & & & \\
N0 & Reference & & \\
N1 & 1.55 & $1.41-1.70$ & $<0.001$ \\
N2 & 2.03 & $1.84-2.25$ & $<0.001$ \\
Grade & & & \\
Well & & & \\
Moderate & Reference & & \\
Poor & 1.39 & $1.21-1.60$ & $<0.001$ \\
Undifferentiated & 1.96 & $1.70-2.26$ & $<0.001$ \\
Radiotherapy (no/unkown vs yes) & 0.81 & $0.74-0.88$ & $<0.001$ \\
Chemotherapy (no/unkown vs yes) & 0.49 & $0.45-0.53$ & $<0.001$ \\
\hline
\end{tabular}

HR: Hazard Ratio; Cl: Confidence Interval
(95\% CI 0.085-0.197), respectively. The IDIs for OS at 1, 2,3 , and 5 years were $0.050,0.063,0.056$, and 0.045 , respectively $(p<0.001)$ (Table 4$)$. The results were also verified in the validation cohort (Table 4). Therefore, the nomogram had better predictive accuracy for OS at 1,2, 3 , and 5 years than the AJCC 8th staging system.

The time-dependent AUCs of the nomogram for predicting OS within 60 months in the training and validation cohorts are shown in Fig. 3A-B. The AUCs were significantly higher in both cohorts than those of the AJCC 8th staging system, indicating that the model had favourable discrimination. The calibration curves for survival probability showed that the nomogram prediction had good consistency with the actual observation in the training cohort at $12,24,36$, and 60 months (Fig. 3C-F); the validation cohort showed similar results (Supplementary Fig. S3).

Based on the optimal cut-off value of the total nomogram score obtained using X-tile, we divided all patients into three risk groups: 'low risk' $(\leq 138)$, 'medium risk' (139-195), and 'high risk' (> 195). The detailed risk score is shown in Supplementary Table S2. The KM survival curves showed that the nomogram risk grouping had better discrimination for OS than the AJCC 8th staging system (Fig. 4, Supplementary Table S3).

\section{Clinical utility analysis of the nomogram}

To explore the clinical utility of the nomogram, we performed DCA. The results showed that the nomogram had better net benefits than the AJCC 8th staging system in both the training and validation cohorts (Fig. 5A-B), suggesting that the clinical utility of the nomogram is better than that of the AJCC 8th staging system.

\section{Discussion}

Few patients with PHAC are able to undergo surgical resection, and even patients who do undergo resection have a low 5-year survival rate. Therefore, there is an urgent need for a nomogram to predict survival for these patients. Since patients with metastatic PHAC are less likely to undergo surgery, we are more interested in patients who undergo resection of non-metastatic PHAC and desired to develop a predictive nomogram for this cohort.

Here, we performed a population-based study involving data from 6419 patients who underwent resection of non-metastatic PHAC from the SEER database. After assessing multiple variables, including age, gender, and AJCC staging, we finally developed a nomogram including five independent prognostic factors ( $\mathrm{T}$ stage, $\mathrm{N}$ stage, grade, radiotherapy, and chemotherapy) that predicted OS at 1,2, 3, and 5 years after resection with high accuracy. Importantly, these prognostic factors are easy to obtain in clinical practice. The results of our analyses, 


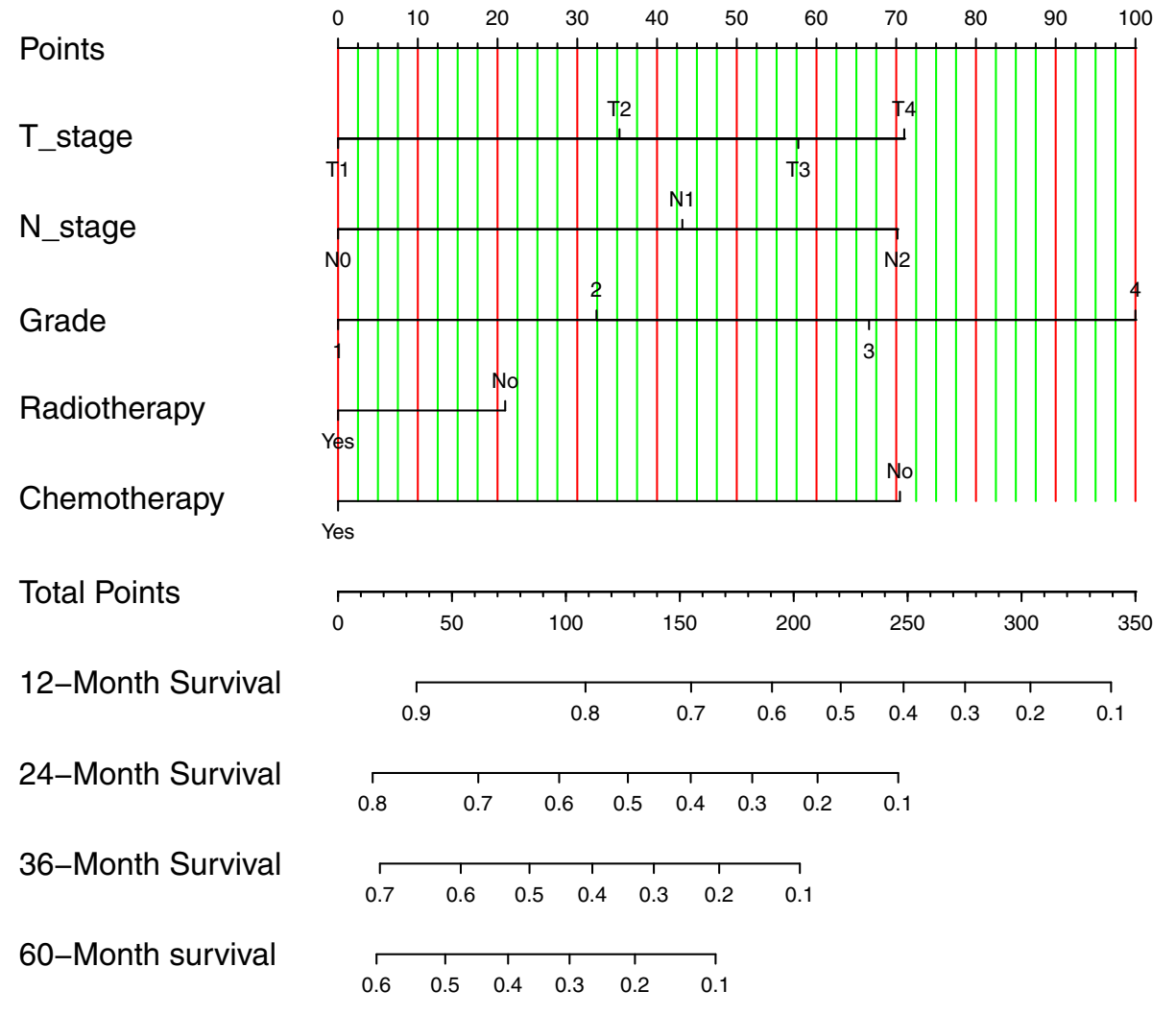

Fig. 2 The nomogram predicting overall survival of patients with non-metastatic PHAC. PHAC: pancreatic head adenocarcinoma

Table 4 The NRIs, IDIs, and C-indexes of the nomogram and AJCC staging system in OS prediction

\begin{tabular}{|c|c|c|c|c|c|c|}
\hline \multirow[b]{2}{*}{ Index } & \multicolumn{3}{|c|}{ Training cohort } & \multicolumn{3}{|c|}{ Validation cohort } \\
\hline & Estimate & $95 \% \mathrm{Cl}$ & $p$ value & Estimate & $95 \% \mathrm{Cl}$ & $p$ value \\
\hline \multicolumn{7}{|c|}{ NRI (vs. the AJCC staging) } \\
\hline For 12-month OS & 0.305 & $0.248-0.357$ & & 0.212 & $0.155-0.332$ & \\
\hline For 24-month OS & 0.227 & $0.172-0.284$ & & 0.163 & $0.104-0.258$ & \\
\hline For 36-month OS & 0.057 & $0.003-0.108$ & & 0.087 & $0.024-0.232$ & \\
\hline For 60-month OS & 0.132 & $0.085-0.197$ & & 0.186 & $0.089-0.263$ & \\
\hline \multicolumn{7}{|c|}{ IDI (vs. the AJCC staging) } \\
\hline For 12-month OS & 0.05 & & $p<0.001$ & 0.044 & & $p<0.001$ \\
\hline For 24-month OS & 0.063 & & $p<0.001$ & 0.059 & & $p<0.001$ \\
\hline For 36-month OS & 0.056 & & $p<0.001$ & 0.055 & & $p<0.001$ \\
\hline For 60-month OS & 0.045 & & $p<0.001$ & 0.046 & & $p<0.001$ \\
\hline \multicolumn{7}{|l|}{ C-index } \\
\hline The nomogram & 0.676 & $0.665-0.687$ & & 0.667 & $0.649-0.684$ & \\
\hline The AJCC staging & 0.581 & $0.570-0.591$ & & 0.566 & $0.548-0.584$ & \\
\hline Change & 0.095 & & $p<0.001$ & 0.101 & & $p<0.001$ \\
\hline
\end{tabular}

AJCC: American Joint Committee on cancer; OS: overall survival; IDI: integrated discrimination improvement; NRI: net reclassification improvement; C-index: concordance index; Cl: Confidence Interval 

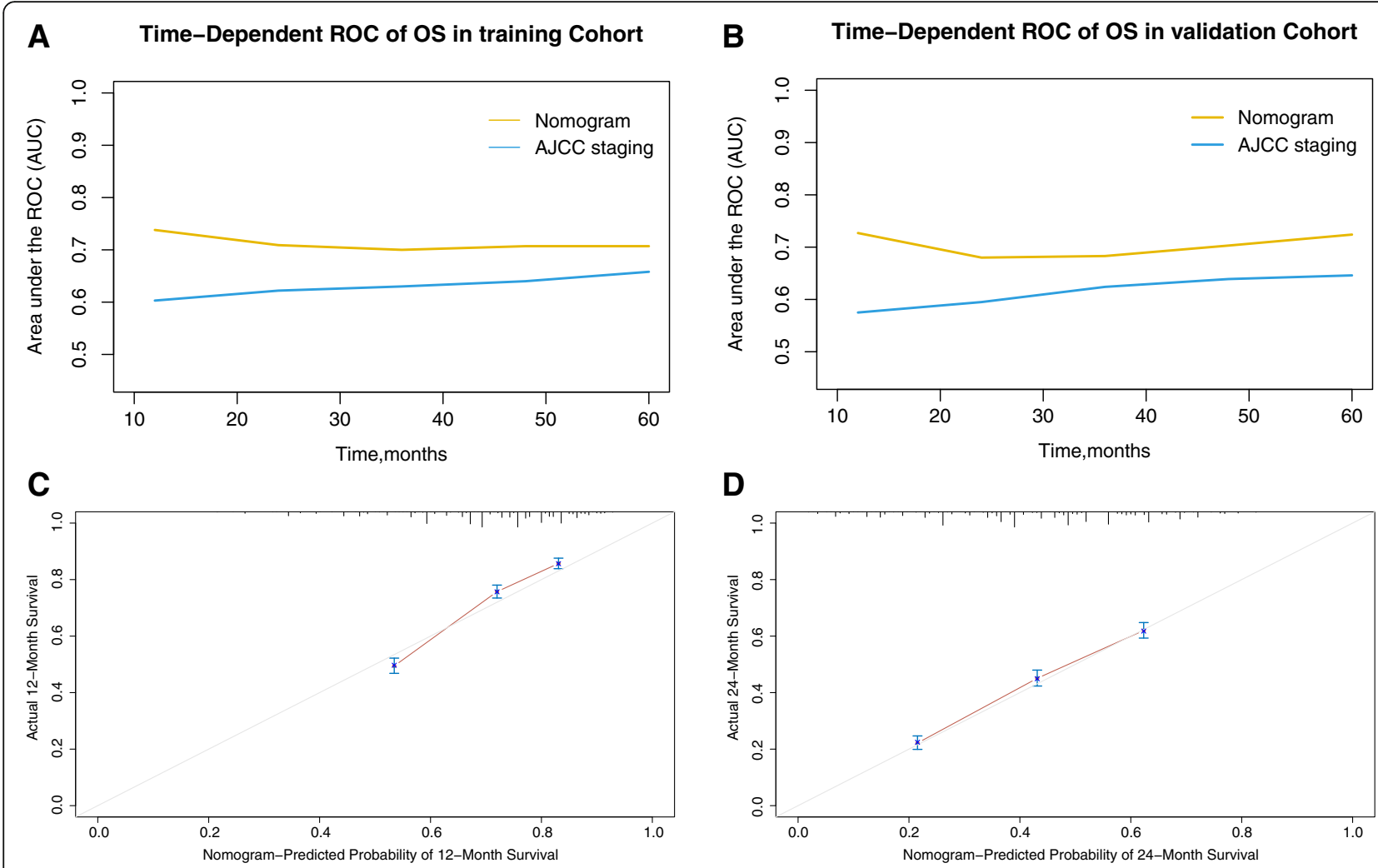

D
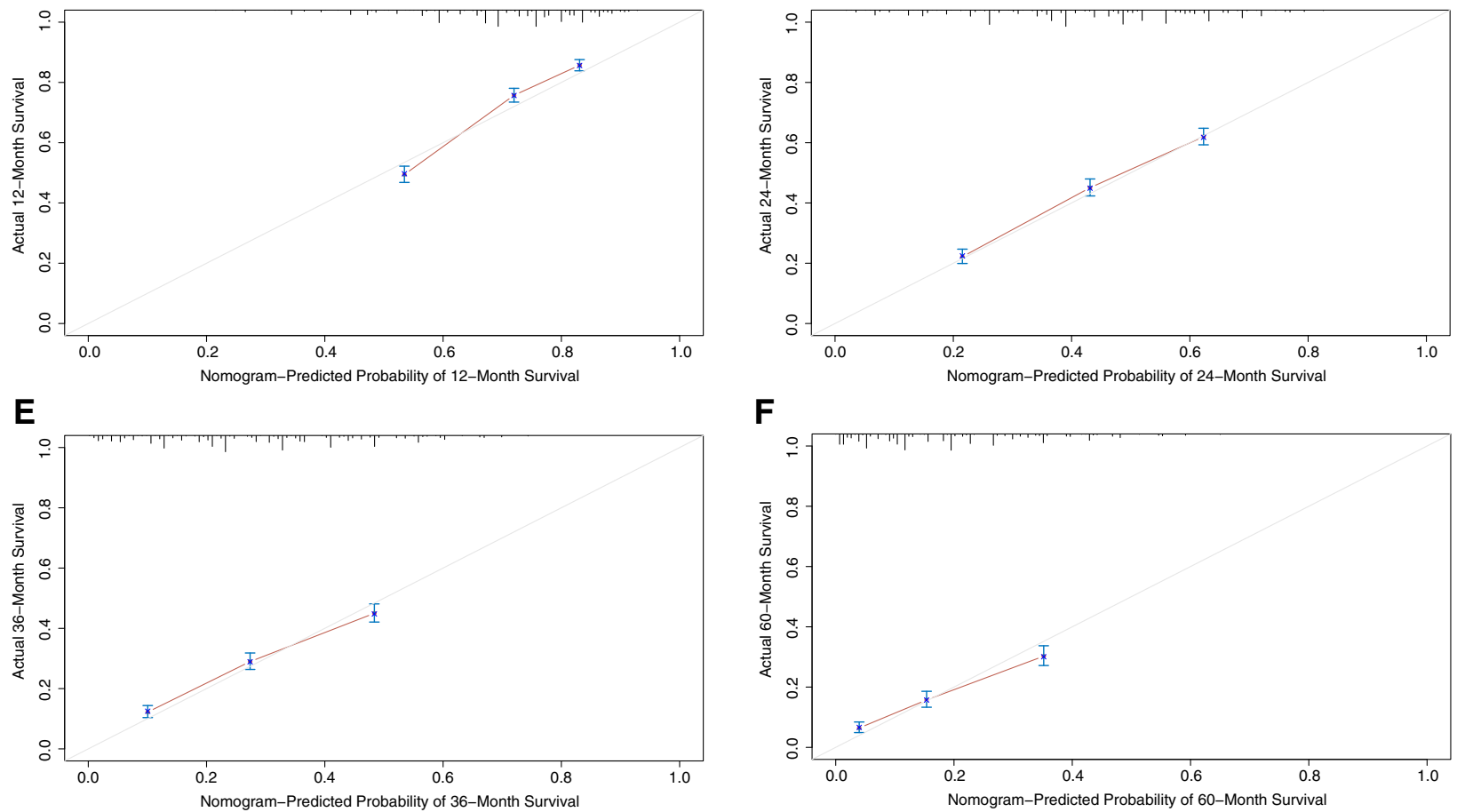

Fig. 3 a-b Comparison of the time-dependent AUCs between the nomogram and the AJCC staging system in the training and validation cohorts. c-f Calibration curves showing the probability of 12-, 24-, 36-, and 60-month overall survival of the model prediction and the observed survival in the training cohort. AUC: area under the curve; AJCC: American Joint Committee on Cancer

including the AUC, NRI, and IDI, showed that our model had better accuracy for predicting survival than the AJCC 8th staging system, and DCA showed the model had higher clinical benefits than the AJCC staging system. The calibration curves also showed good discrimination and calibration capabilities.

Previous studies have proposed various significant prognostic factors for patients with pancreatic cancer, such as age, lymph node metastasis, and biochemical indicators [10, 12-14]. Similarly, our study also demonstrated that age, $\mathrm{T}$ stage, $\mathrm{N}$ stage, grade, the LNR, and tumour size were significant risk factors; radiotherapy and chemotherapy were strong protective factors. He et al. revealed that high-grade classification indicates poor prognosis in adenocarcinoma of the pancreatic body and tail [11]. In our study, the high-grade classification was a strong prognostic risk factor of PHAC, so we included tumour grade into this model. The LNR was recently implemented as a more accurate assessment of lymph node metastasis to modify $\mathrm{N}$ stage and has been used to predict prognosis and guide clinical practice for patients with pancreatic cancer [15-18]. In our study, the LNR was an independent prognostic factor in Cox regression analysis. The cut-off value of 0.2 

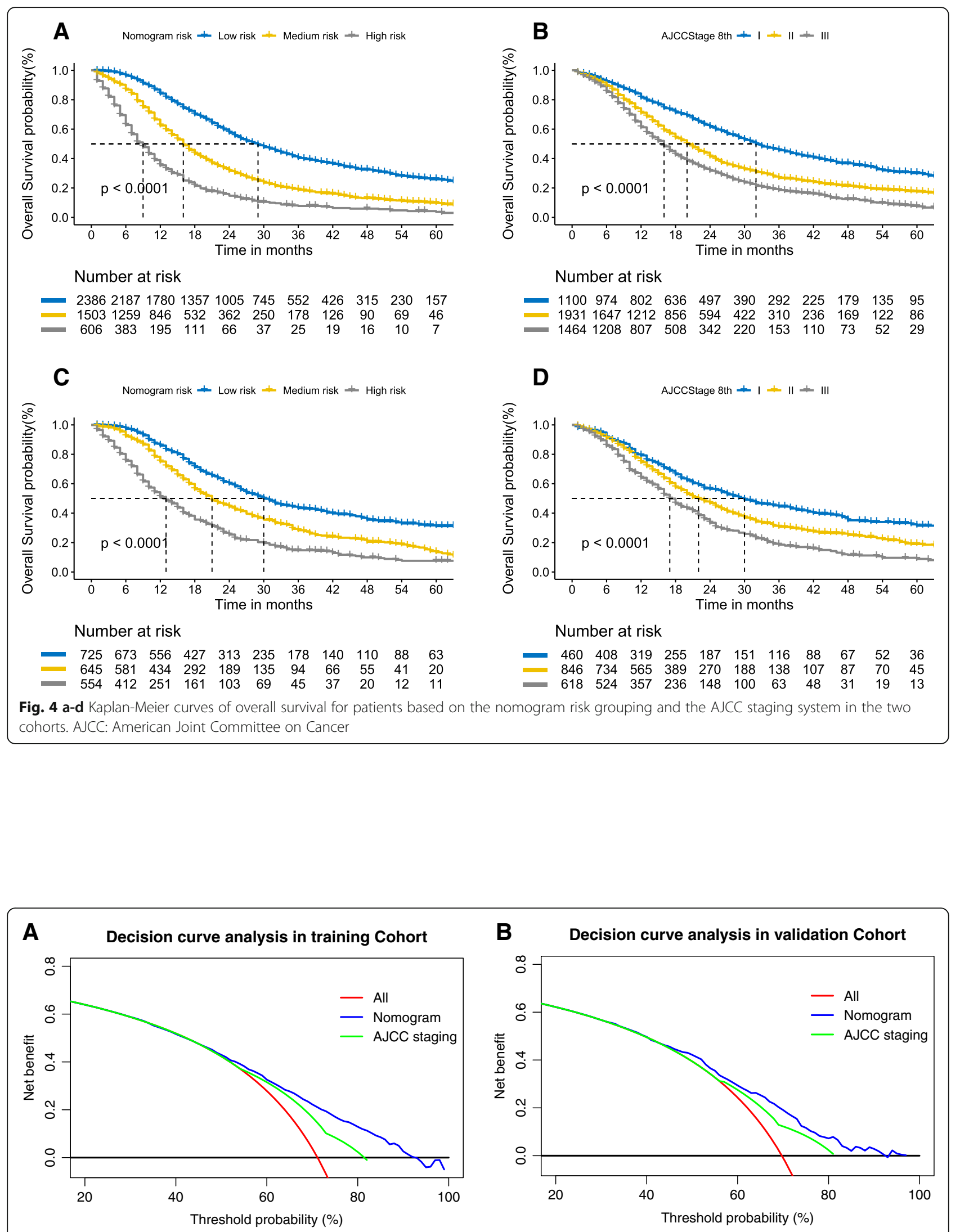

Fig. 5 a-b Decision curve analysis showed clinical benefits of the model predicting overall survival in the training and validation cohorts 
was also appropriate and further applied to the controversial problem of threshold. We then further compared the predictive abilities of the LNR and $\mathrm{N}$ stage by comparing their $\mathrm{C}$-indexes. Interestingly, we found no significant difference. However, $\mathrm{N}$ stage is a more accessible factor than the LNR. Therefore, the LNR may not be superior to $\mathrm{N}$ stage for predicting survival in patients with non-metastatic PHAC after surgery.

Radical surgery and chemoradiotherapy are currently the main treatment strategies for patients with pancreatic cancer [1]. A phase III clinical trial revealed that adjuvant chemoradiotherapy can significantly increase the R0 resection rate and prolong survival [19]. Recent studies have also reported that chemoradiotherapy is a key protective factor in advanced pancreatic cancer [20-22]. Administration of adjuvant chemoradiotherapy can significantly improve the survival of PHAC patients. In our study, we demonstrated that radiotherapy and chemotherapy were strong protective factors in this large study population. Therefore, our nomogram included these interventions. The nomogram had better predictive capability than the AJCC 8th staging system. Therefore, physicians can use postoperative indicators and these treatment measures to assess patient prognosis and guide further treatment.

Although our model had good performance for predicting survival, some limitations must also be noted. First, the data were collected from the SEER database, and this was a retrospective study with inherent defects. Further, some significant prognostic factors, such as the resection margin, the level of carbohydrate antigen 199, and some physical indices, were not available in the SEER database. Second, the cut-off value of partial variables in our study were calculated by X-tile, and the wide application of the threshold requires further confirmation. Further large prospective studies to confirm the effectiveness of our prognostic model are also needed.

\section{Conclusions}

We developed and validated a nomogram that has high accuracy in predicting OS in patients after resection of non-metastatic PHAC. All of the included prognostic factors are easy to obtain. This nomogram could promote the development of a novel staging system and assist clinicians in conducting personalized clinical treatment.

\footnotetext{
Abbreviations

AJCC: American Joint Committee on Cancer; AUC: Area under the curve; Cindex: Concordance index; Cl: Confidence Interval; DCA: Decision curve analysis; HR: Hazard Ratio; OS: Overall survival; ICD: International Classification of Diseases; IDI: Integrated discrimination improvement; KM: Kaplan-Meier; LNR: Lymph node ratio; NRI: Net reclassification improvement; PHAC: Pancreatic head adenocarcinoma; SEER: Surveillance, Epidemiology, and End Results database
}

\section{Supplementary Information}

The online version contains supplementary material available at https://doi. org/10.1186/s12885-021-08250-4.

Additional file 1: Fig. S1. Cut-off value of the LNR and tumor size were calculated from the training cohort. A, B LNR. C, D tumor size. In the plot $A$ and $C$, red represents the negative correlation between the variables and survival, whereas green represents a positive correlation. In the plot $B$ and $C$, it shows the cut-off value of variables and numbers of patients in subgroups. Fig. S2. Kaplan-Meier curves of patients based on obtained prognostic variables. A age. B race. C sex. D T stage. E N stage. F grade classification. G LNR. H tumor size. I radiotherapy. J chemotherapy. Fig. S3. Calibration curves showed the probability of 12-, 24-, and 36-, and 60- year OS between the model prediction and the practical observation in the validation cohort. Table S1. The optimal cut-off value of LNR and tumor size in training cohort. Table S2. Nomogram score of prognostic variables. Table S3. Overall Survival probability and Median Survival Time of Prognostic Nomogram Score and current criteria in the two cohorts.

\section{Acknowledgements}

We are grateful for the registration of SEER program for the creation of the SEER database.

\section{Authors' contributions}

WBZ, ZZW and FW contributed equally to this work, and were considered as the co-first authors. WBZ, ZZW and FW participate in design and conception of this study. GZ collected the data from the database. All authors participated in writing the manuscript, while RL proposed the conception and revised the manuscript. All authors read and approved the final manuscript.

\section{Funding}

Not applicable.

\section{Availability of data and materials}

The data that support the findings of this study are available from the corresponding author upon reasonable request, and were publicly available and obtained from the Surveillance, Epidemiology, and End Results database (http://seer.cancer.gov/).

\section{Declarations}

Ethics approval and consent to participate

No applicable.

\section{Consent for publication}

Consent for publication was obtained from all authors.

\section{Competing interests}

The authors declare that they have no conflicts of interest related to this manuscript.

\section{Author details}

${ }^{1}$ Faculty of Hepato-Pancreato-Biliary Surgery, Chinese PLA General Hospital, No.28 Fuxing Road, Haidian District, Beijing 100853, China. ${ }^{2}$ Institute of Hepatobiliary Surgery of Chinese PLA, Beijing, China. ${ }^{3}$ Key Laboratory of Digital Hepetobiliary Surgery PLA, Beijing, China.

Received: 6 March 2021 Accepted: 23 April 2021

Published online: 08 May 2021

References

1. Mizrahi JD, Surana R, Valle JW, Shroff RT. Pancreatic cancer. Lancet. 2020; 395(10242):2008-20. https://doi.org/10.1016/S0140-6736(20)30974-0.

2. Zhu H, Li T, du Y, Li M. Pancreatic cancer: challenges and opportunities. BMC Med. 2018;16(1):214. https://doi.org/10.1186/s12916-018-1215-3.

3. Neoptolemos JP, Kleeff J, Michl P, Costello E, Greenhalf W, Palmer DH. Therapeutic developments in pancreatic cancer: current and future perspectives. Nat Rev Gastroenterol Hepatol. 2018;15(6):333-48. https://doi. org/10.1038/s41575-018-0005-x. 
4. McGuigan A, Kelly P, Turkington RC, Jones C, Coleman HG, McCain RS Pancreatic cancer: a review of clinical diagnosis, epidemiology, treatment and outcomes. World J Gastroenterol. 2018;24(43):4846-61. https://doi.org/1 0.3748/wjg.v24.i43.4846.

5. Seufferlein T, Hammel P, Delpero JR, Macarulla T, Pfeiffer P, Prager GW, et al. Optimizing the management of locally advanced pancreatic cancer with a focus on induction chemotherapy: expert opinion based on a review of current evidence. Cancer Treat Rev. 2019;77:1-10. https://doi.org/10.1016/j. ctrv.2019.05.007

6. Stathis A, Moore MJ. Advanced pancreatic carcinoma: current treatment and future challenges. Nat Rev Clin Oncol. 2010;7(3):163-72. https://doi.org/10.1 038/nrclinonc.2009.236.

7. Lou X, Li J, Wei YQ, Jiang ZJ, Chen M, Sun JJ. Comparable prevalence of distant metastasis and survival of different primary site for $L N+$ pancreatic tumor. J Transl Med. 2020;18(1):266. https://doi.org/10.1186/ s12967-020-02438-1.

8. MB A, American joint committee on Cancer, American Cancer Society. AJCC Cancer staging manual. In: American Joint Committee on Cancer. 8th ed. Chicago: Springer; 2017

9. Sheng W, Dong M, Wang G, Shi X, Gao W, Wang K, et al. The diversity between curatively resected pancreatic head and body-tail cancers based on the 8th edition of AJCC staging system: a multicenter cohort study. BMC Cancer. 2019;19(1):981. https://doi. org/10.1186/s12885-019-6178-Z.

10. Zou Y, Han H, Ruan S, Jian Z, Jin L, Zhang Y, et al. Development of a Nomogram to predict disease-specific survival for patients after resection of a non-metastatic adenocarcinoma of the pancreatic body and tail. Front Oncol. 2020;10:526602. https://doi.org/10.3389/fonc.2020. 526602.

11. He C, Sun S, Zhang Y, Lin X, Li S. Score for the overall survival probability of patients with pancreatic adenocarcinoma of the body and tail after surgery: a novel Nomogram-based risk assessment. Front Oncol. 2020;10:590. https:// doi.org/10.3389/fonc.2020.00590.

12. Midha S, Chawla S, Garg PK. Modifiable and non-modifiable risk factors for pancreatic cancer: a review. Cancer Lett. 2016;381(1):269-77. https://doi. org/10.1016/j.canlet.2016.07.022.

13. Okada K, Murakami Y, Kondo N, Uemura K, Nakagawa N, Seo S, et al. Prognostic significance of lymph node metastasis and micrometastasis along the left side of superior mesenteric artery in pancreatic head Cancer. J Gastrointest Surg. 2019;23(10):2100-9. https://doi.org/10.1007/ s11605-019-04359-x.

14. Tsai S, George B, Wittmann D, Ritch PS, Krepline AN, Aldakkak M, et al, Importance of normalization of CA19-9 levels following Neoadjuvant therapy in patients with localized pancreatic Cancer. Ann Surg. 2020;271(4): 740-7. https://doi.org/10.1097/SLA.0000000000003049.

15. You MS, Lee SH, Choi YH, Shin BS, Paik WH, Ryu JK, et al. Lymph node ratio as valuable predictor in pancreatic cancer treated with $\mathrm{RO}$ resection and adjuvant treatment. BMC Cancer. 2019;19(1):952. https://doi.org/10.1186/s12 885-019-6193-0

16. Tol JA, et al. Impact of lymph node ratio on survival in patients with pancreatic and periampullary cancer. Br J Surg. 2015;102(3):237-45. https:// doi.org/10.1002/bjs.9709.

17. Hartwig W, Hackert T, Hinz U, Gluth A, Bergmann F, Strobel O, et al. Pancreatic cancer surgery in the new millennium: better prediction of outcome. Ann Surg. 2011;254(2):311-9. https://doi.org/10.1097/SLA.0b013 e31821fd334.

18. Berger AC, Watson JC, Ross EA, Hoffman JP. The metastatic/examined lymph node ratio is an important prognostic factor after pancreaticoduodenectomy for pancreatic adenocarcinoma. Am Surg. 2004 70(3):235-40 discussion 240

19. Versteijne E, Suker M, Groothuis K, Akkermans-Vogelaar JM, Besselink MG, Bonsing BA, et al. Preoperative Chemoradiotherapy versus immediate surgery for Resectable and borderline Resectable pancreatic Cancer: results of the Dutch randomized phase III PREOPANC trial. J Clin Oncol. 2020;38(16):1763-73. https://doi.org/1 $0.1200 / J C O .19 .02274$

20. Liu JH, Wang SF, Li BR, Zhu X. Development and validation of an individualized nomogram for predicting pancreatic adenocarcinomaspecific survival: a SEER population analysis of 5,805 patients. Eur Rev Med Pharmacol Sci. 2020;24(20):10483-95. https://doi.org/10.26355/ eurrev_202010_23401.
21. Krishnan S, Chadha AS, Suh Y, Chen HC, Rao A, Das P, et al. Focal radiation therapy dose escalation improves overall survival in locally advanced pancreatic Cancer patients receiving induction chemotherapy and consolidative Chemoradiation. Int J Radiat Oncol Biol Phys. 2016;94(4):75565. https://doi.org/10.1016/j.jirobp.2015.12.003.

22. Mas L, Schwarz L, Bachet JB. Adjuvant chemotherapy in pancreatic cancer: state of the art and future perspectives. Curr Opin Oncol. 2020;32(4):356-63. https://doi.org/10.1097/CCO.0000000000000639.

\section{Publisher's Note}

Springer Nature remains neutral with regard to jurisdictional claims in published maps and institutional affiliations.

\section{Ready to submit your research? Choose BMC and benefit from:}

- fast, convenient online submission

- thorough peer review by experienced researchers in your field

- rapid publication on acceptance

- support for research data, including large and complex data types

- gold Open Access which fosters wider collaboration and increased citations

- maximum visibility for your research: over $100 \mathrm{M}$ website views per year

At BMC, research is always in progress.

Learn more biomedcentral.com/submissions 